\title{
ON THE NOTION OF EFFECTIVE IMPEDANCE
}

\author{
ANNA MURANOVA
}

Abstract. It is known that electrical networks with resistors are related to the Laplace operator and random walk on weighted graphs. In this paper we consider more general electrical networks with coils, capacitors, and resistors. We give two mathematical models of such networks: complex-weighted graphs and graphs with weights from the ordered field of rational functions. The notion of effective impedance in both approaches is defined.

Mathematics subject classification (2010): 05C22, 05C25, 34B45, 39A12, $12 \mathrm{~J} 15$. functions.

Keywords and phrases: Weighted graphs, electrical network, Laplace operator, ordered field of rational

\section{REFERENCES}

[1] O. BRUNE, Synthesis of a finite two-terminal network whose driving-point impedance is a prescribed function of frequency, Thesis (Sc. D.). Massachusetts Institute of Technology, Dept. of Electrical Engineering, Massachusetts, 1931.

[2] Charles A. Desoer, Ernest S. Kuh, Basic circuit theory, McGraw-Hill Book Company, USA, 1969.

[3] P.G. Doyle, J.L. SNell, Random walks and electric networks, Carus Mathematical Monographs 22, Mathematical Association of America, Washington, DC, 1984.

[4] Richard P. Feynman, Robert B. Leighton, Matthew Sands, The Feynman lectures on physics, Volume 2: Mainly Electromagnetism and Matter, Addison-Wesley publishing company, Reading, Massachusetts, Fourth printing - 1966.

[5] A. GRIGOR'YAN, Introduction to Analysis on Graphs, AMS University Lecture Series, Volume: 71, Providence, Rhode Island, 2018.

[6] Edward Hughes, Electrical and electronic technology, Pearson Education Limited, England, Tenth edition -2008.

[7] David A. Levin, Yuval Peres, Elizabeth L. Wilmer, Markov Chains and Mixing Times, AMS University Lecture Series, Providence, Rhode Island, 2009.

[8] ARIEH L. Shenkman, Circuit analysis for power engineering handbook, Springer Science+Business Media, B. V, USA, 1998.

[9] B. L. VAN DER WAERden, Algebra, Volume I, Springer, New York, 2003. 